and definite foundation in a much disturbed ground.

Into this bewildering maze of circumstances there has been flung, with considerable force recently, the challenge of synthetic oil from hydrogenation process. What is the significance of this incursion? It is inconceivable, aside from all economic argument, that this factor in the situation can be other than one to be seriously reckoned with; it is disturbing even now ; it is bound to be formidable ultimately in the petroleum industry, and to have far-reaching consequences on the technical operations involved therein.

The history of chemical industry is rich in examples of new processes in which the established commercial success was achieved among the ruins of discarded doubts and scepticisms at the outset. Hydrogenation is a long-tried process ; it has, as a technical procedure, been brought to a high state of efficiency. It is chemical engineering at its best. Oil production from coal, whether by this or by any other means is, and must inevitably be, on whatever scale achieved, a product of chemical industry. To this extent, the product differs fundamentally from anything realised by the petroleum industry.

The coal industry per se is non-existent in the same sense in which we contemplate and assess the activities of the oil industry to-day. It discarded individuality long ago in favour of secondhand recognition through the medium of dependent products such as gas, coal-tar, dyes and allied substances, the enormous developments of which not only built up relevant commercial activities, but also have contributed more to the growth of chemical industry as a whole than probably any other branch of it. Hydrogenated coal-oil when it arrives as an accepted economic commodity will simply add another weapon to the already powerful array of forces which chemistry commands in the sway of modern civilisation.

The moral to the petroleum industry in general, and to the technology in particular, is surely obvious. There has for long been the broadest possible overlap between research within the petroleum and chemical industries, at least as technically interpreted, and where basic substances common to both have been involved. Research, temporarily retarded as it may have been by prevalent conditions, has none the less forged ahead in chemical industry, as keenly animated as ever by the desire to conquer fresh fields and produce new outlets for natural resources at the present time only partially or extravagantly exploited. The trend of petroleum technology, if it is to compete with the incidence of an independent and biased chemical industry, must, apart from the actual winning of oil, inevitably lead in the direction of finding new and extended uses for its raw material and basic products.

It is insufficient to point to a succession of highly refined products of crude oil such as petroleum, paraffin, lubricating and fuel oils, wax and asphaltic bitumen-the high standard of production of which to-day is freely acknowledged -as though these represent monuments to final achievement. There can be no such finality, and research must aim far beyond the mere improvement of technique of production, the multiplicity of types and qualities of distillates, and the goal of supplying markets with what they have been accustomed.

In point of fact, the domain of petroleum technology is no longer inviolable; and if it does not of its own initiative expand actively into the realm of chemical industry, then assuredly will the latter gradually stretch the tide-mark of overlap until the merger is complete.

Petroleum, like coal, is a wasting asset. It is no longer the prerogative of the oil industry alone and, as a natural resource still possessing immense latent possibilities of more efficient development, it must come into line with other basic raw materials and be subject to that vigorous independent investigation which is afforded by intensive chemical research. Only in this way will it avoid some of the calamitous happenings which have led to the acute problems of efficient coal exploitation and utilisation, for which, it is to be noted, chemical industry has gone a long way to find a practical and economic solution. H. B. M.

\section{Age of Retirement in India}

$\mathrm{U}$ NDER the title "The Fifty-five Year Rule", the November issue of the Indian monthly, Current Science, contains a long leading article advocating the abolition of retirement from educational posts at the age of fifty-five, as is now compulsory under the fundamental rules, and the raising of the age limit to sixty years in the first instance, or making such appointments for life. We have read the article with some surprise, since we are convinced that the concensus of opinion of 
those engaged in educational work in Great Britain regards the comparatively recent introduction of the Universities' Superannuation Scheme with its corollary, a compulsory age of retirement, as a distinct contribution both to the efficiency of the universities and of the schools.

Although it is not uncommon for men of mature age to make valuable direct contributions to knowledge, there is no doubt that with increasing years their teaching, whether it be in the school class room or the university lecture theatre, tends to become stereotyped and lacking in inspiration. Furthermore, they show less and less inclination to initiate young students into the methods of research, although they themselves may continue to be actively engaged therein. A not unimportant feature of the superannuation scheme has been to accelerate the promotion of the younger men and so stimulate employment. So far as we can gather, the main objection to compulsory retirement is that it deprives the university of the services of men of mature experience, but we doubt if this is actually so in practice. It is now almost invariably the custom to confer upon the retiring professor the title of emeritus, which is recognised as carrying with it the right to laboratory accommodation in his old department, a privilege of which advantage is frequently taken.

The writer of the article in Current Science appears to be under the impression that professorships in European universities are life appointments. As a matter of fact, there remain a few professorships in Scottish Universities and at Oxford and Cambridge where a definite age of retirement has not been assigned, but as these fall vacant the general practice of retirement at sixty is being adopted. It is true that the German universities do not fix a date for retirement, but in that country a professor's pension is the same as his salary, and consequently there is not the same temptation as there is in Great Britain and in India to continue in office after effective service can be rendered.

It is suggested in the article that, as the result of the great advances made in preventive medicine, retirement at the early age of fifty-five is no longer necessary in India. Whilst the increased security of life in the East might be used as an argument for raising the retiring age to sixty, we doubt whether this is actually the case. Climate cannot be altered. The Indian matures much earlier than the European and he ages correspondingly earlier, so that as a general rule the present retiring age seems to be comparable to that prescribed in England.
It is pointed out in the article that though the Government has decided to terminate the services of professors and others in the educational service at fifty-five years of age, this is not the practice in the judiciary or administrative posts of cabinet rank. Though this suggests unjust inequality of treatment, we think the conditions are different in the two groups. One of the main essentials for success in education is originality, a gift more common in youth than in age ; this qualification is scarcely required on the Bench. In the field of higher administration we note a growing tendency to find room for the younger men, and we think this is likely to increase in the future.

While we agree with the view expressed in the article that a man is not necessarily disqualified physically or mentally for further useful service in India when he reaches the age of fifty-five, we believe it is not usually desirable for Europeans to remain at work in that country beyond that age. If, however, a university finds it to the advantage of a department to retain a teacher after the age of fifty-five, a man who is still active at that age ought to be able to expect to have his period of service extended to the age of sixty. It is the practice in some educational institutions to make these extensions by one year at a time, and we suggest that if the universities could extend the services by such a period, not exceeding five years in all, after considering the merits of each case, there could be little cause of complaint.

\section{Evolution and Mechanism of Hearing}

Hearing in Man and Animals. By R. T. Beatty. Pp. xi+227. (London : G. Bell and Sons, Ltd., 1932.) 12s. net.

7 HE study of the evolution of the sense-organs has a fascination for the general reader as well as the scientific worker. Our knowledge of the world around us is dependent on the reaction between various forms of physical energy and the appropriate sense organs. Some of the latter, such as touch, smell, taste and sight, are common to all vertebrates and are found in much lower forms of life, but hearing is relatively uncommon and may be regarded as a 'recent' development. In many of the lower forms of life, for example, in insects and fishes, the sense of hearing is either entirely absent or exists in a most rudimentary form.

For its proper understanding, audition requires a knowledge of anatomy, physiology, physics and 